\title{
AN EXPLORATORY STUDY COMPARING CAD TOOLS AND WORKING STYLES FOR IMPLEMENTING DESIGN CHANGES
}

\author{
Phadnis, Vrushank Shripad (1); Leonardo, Kevin Alfonso (1); Wallace, David Robert (1); \\ Olechowski, Alison Louise (2)
}

1: Massachusetts Institute of Technology; 2: University of Toronto

\begin{abstract}
This paper presents the findings of a preliminary study comparing implementation of design changes using various computer-aided design (CAD) working styles. Our study compares individuals' and pairs' completion of a series of changes to a toy car CAD model. We discuss the results in terms of productivity and value added ratio, derived from time-based quantitative data. We also discuss qualitative findings acquired through post-study surveys. Overall, our findings suggest that pairs were less efficient than individual designers due to overheads like communication, history dependency and complex couplings within the CAD model tree. However, it is also noteworthy that within each pair the lead participant's performance was at par with individual participants. Lastly, we also discuss behaviors and patterns that emerge as unique to the synchronous collaborative environment, motivating future work.
\end{abstract}

Keywords: Computer Aided Design (CAD), Collaborative design, Online CAD, Design process, Design management

\section{Contact:}

Phadnis, Vrushank Shripad

Massachusetts Institute of Technology

Mechanical Engineering

Canada

vphadnis@mit.edu 


\section{INTRODUCTION}

Designing using Computer-Aided Design (CAD) software has always involved collaboration. Topdown assembly and master modeling techniques are the norm in industry and have proven to be efficient at organizing CAD modelling. In this approach, the engineering product to be modeled is hierarchically decomposed until the modeling tasks are at an appropriate size for a single designer to work on. Then the designers mostly work by themselves in silos. This top-down architecture drives the relationships between parent and subassembly/ children parts (Libardi et al., 1988). This structured approach to CAD work distribution has been well established but has its set of limitations which includes a fragmented product design process.

The potential of collaborative features in synchronous CAD is intriguing given that design is a social process that involves multiple people working towards a solution (Bucciarelli and Kuhn, 1997). More products are being designed by global teams and high demand for anytime availability of product data has become a compelling CAD software requirement.

Recent cloud computing capabilities have made web-based synchronous CAD commercially available at scale. The ability to have multiple designers simultaneously modifying a model from their own workstations is now a reality through cloud-based software offerings (Andreadis et al., 2015; Li et al., 2005; Wu et al., 2017). A synchronous CAD platform offers numerous benefits like: synchronous access, cost effectiveness, higher utilization of resources, and enhanced security (Li et al., 2005; Woo, 2014). However, these benefits are visible at the organization level only after all components of the product development cycle are brought online (Woo, 2014). There are still questions to be answered surrounding design in the cloud related to usability, security, and computational performance (Wu et al., 2017).

We are yet to uncover the effects of collaborative CAD tools on underlying design processes at designer (user) level, or team level, and how to best make use of their enhanced feature set. We anticipate that working in a fully synchronous collaborative CAD environment will affect key design metrics like creativity, communication fluency, design quality, productivity and efficiency. To narrow the scope of our work, we aim to elucidate effects on time-based metrics like value added ratio and productivity from working on fully-synchronous collaborative CAD work.

In this paper we present the results of an exploratory study to better understand the dynamics at play during fully-synchronous collaborative design, and how they might compare to design using traditional single-user CAD software. First, we briefly review the relevant literature on collaborative design work, in particular collaboration using synchronous CAD design tools. Next, we describe the set-up of our exploratory experiment. Then we present preliminary time-based results of these experiments and discuss implications of our findings. Finally, we describe future areas of research motivated by these initial findings.

\section{RELATED WORK}

A limited number of previous studies have aimed to investigate collaborative CAD teams, some even using synchronous collaborative CAD tools.

Eves et al. (2018) developed their own collaborative computer-aided design software, Multi-user CAD (MUCAD) which allows synchronous design. This enabled a comparison of the output of four-person teams using MUCAD and traditional CAD (sharing models through email). This study found that use of MUCAD increases the awareness of teammates' activities, and communication between team members. However, no statistically significant findings were found related to model quality or team productivity, potentially due to limitations of a small sample size. It is important to note that the MUCAD software in this study was under development, a limitation for this work and on the quality of the collected data. The authors note anecdotally that multi-user teams appeared to have better interface management, and a better sense of current state of model and what still needed to be done.

Two other studies have looked for associations between designer teams or product-type and design behaviors using full-synchronous collaborative CAD tools. Stone et al. (2017) set out to establish a method to determine the optimal number of designers on a part based on characteristics of the part itself and the architecture of its features. Teams varied in size from one- to four-person. No statistically significant trends were found. The authors had to adjust performance to compensate for the impact of software bugs, which is an important limitation of this work to consider. Another study 
looked at various team-member dynamics, especially communication, during a MUCAD design competition of three-person teams (Stone, Salmon, Eves et al., 2017). Teams that encouraged effective forms of communication and teams whose members scored similarly on Purdue Spatial Visualization Test (a test of spatial manipulation ability) performed better than other teams. The authors compared audio recordings, and posited that patterns of communication could provide important insight. The authors noted that anecdotally, high-performing teams tended to communicate less.

Holyoak et al. (2014) presented an approach for collaborating on fully-synchronous CAD software. This proposed design process provides guidance based on inputs of design specifications and task distribution (analyzed for dependence via a design structure matrix), aiming to take advantage of the ability for multi-users to work concurrently in parallel. By analyzing the team for expertise and decision-making authority, the output of the design process was a list of tasks and corresponding personnel groups to accomplish those tasks. The authors claimed that their process has the potential to reduce wait times and iterations, and therefore overall design time. The authors presented a small data set comparing teams of three who follow this new process to teams of three who iterate through a design, indicating that the multi-user teams following the new process finish design tasks more quickly and with more specifications satisfied. However, these results were not statistically examined for significance, likely due to the small number of trials.

Current research specific to fully synchronous collaborative CAD has been limited by buggy-software and small sample sizes, yet makes important observations of trends in behavior and outcomes. These works highlight how little is known about synchronous collaborative CAD, and inspires potential directions for future detailed study.

We are now at a point where technical challenges are no longer barriers to fully synchronous CAD implementation. This paper aims to present further exploratory work to examine differences between traditional CAD and synchronous CAD. In this work, we use a commercially available cloud-based CAD software Onshape. The small sample size of our participant pool is a limitation and prevents us from attaining statistically significant results. However, this work uncovers insights to inform future work leading to a larger follow-on study. We seek a baseline understanding of the differences between working styles, and more specifically benchmark the working styles on the metric of productivity and value added ratio.

\section{EXPERIMENT APPROACH}

\subsection{Design and setup}

Our study was setup to compare three distinct CAD working styles: single person operating on traditional CAD, single person operating on synchronous CAD, and lastly teams of two operating on synchronous CAD. We chose to study pairs as against larger teams to simplify our analysis and to mitigate effects from group dynamics. All study trials are summarized in Figure 1 below. We created multiple configurations of paired working styles to get insight into the influence of communication and differing skill levels on synchronous CAD work. We chose SolidWorks as our traditional CAD platform and Onshape as our synchronous CAD alternative because both programs present a similar feature set within the constraints of our study. Both programs offer a stable CAD environment and are commercially used in industry. In the single user working styles, an individual participant worked by themselves. In the paired working styles, the participants shared a common CAD environment which meant they could see each other's work. Communication access was withheld from one of the pairs, whereas the other pairs were able to use the built-in chat feature. In another pairing, we partnered a novice CAD user alongside a baseline participant. Our study protocol was approved by the Committee on the Use of Humans as Experimental Subjects at Massachusetts Institute of Technology (MIT). In compliance, we have adopted the following participant name coding: OS for Onshape participant, SW for SolidWorks participant, BP-1 for first baseline pair participant, BP-2 for second baseline pair participant, $\mathrm{NC}-1$ for first no communication participant, $\mathrm{NC}-2$ for second no communication participant, NB-B for baseline participant, NB-N for novice participant. The goal of these experimental settings was to compare individual CAD work to paired CAD work and then explore the effect of communication and skill mismatch between pairs working on synchronous CAD. The Baseline pair was selected such that each member had similar amount of CAD experience and were provided access to chat communication. 


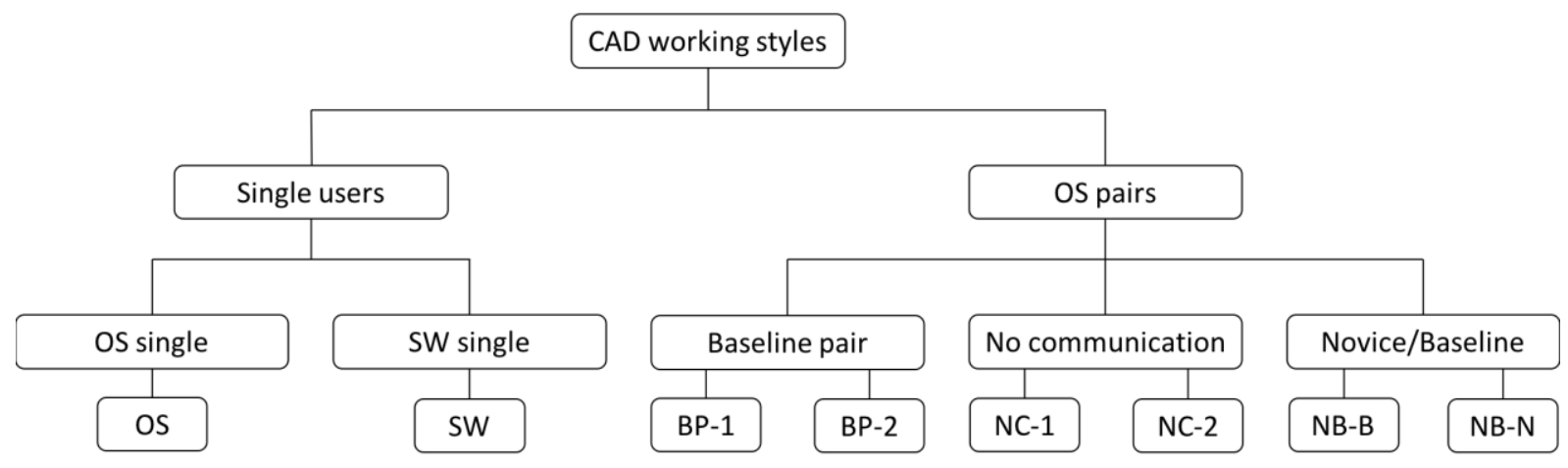

Figure 1. Tree diagram showing various study settings (OS: Onshape, SW: SolidWorks)

The study was conducted in a controlled environment at the MIT Behavioral Research Lab (BRL) where all participants were provided with identical equipment which included workstations with preinstalled CAD software. One such study session in progress is shown in Figure 2c. All studies were performed at BRL in a similar setting.

Every synchronous CAD participant was given a 15-minute demo of unique features of synchronous CAD and were shown the software's communication mechanism.
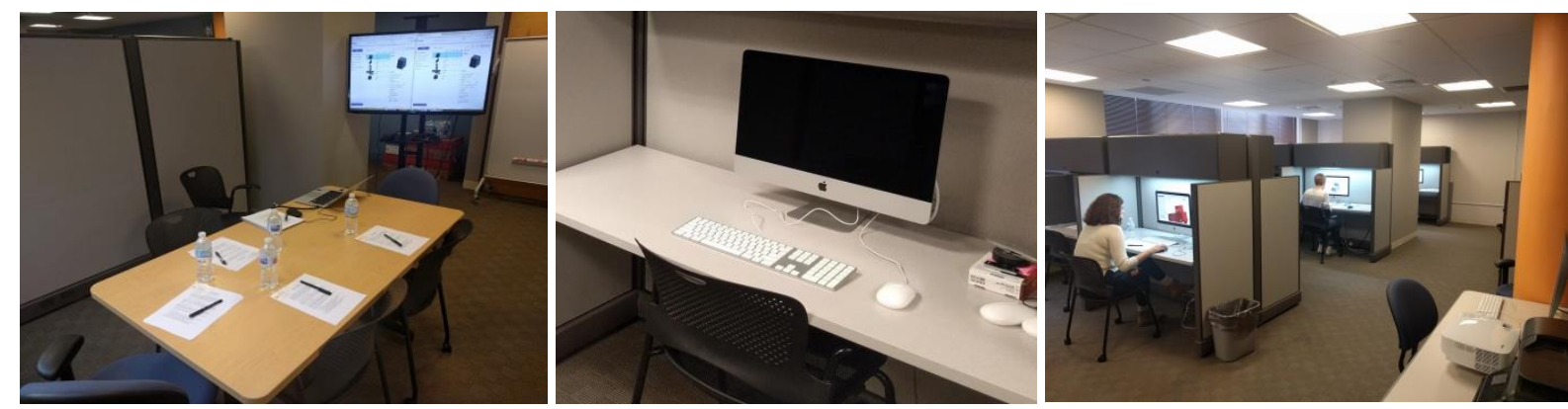

Figure 2. Study setting (ordered left to right): (a) 15 min. demo (b) Typical CAD station

(c) Study in progress at MIT BRL

The study was setup for the participants to role-play a toy designer at a company that was working on an early stage concept for a toy car (shown below in Figure 3). This design artifact was chosen as it was simple, common, and relatable to the participant pool.
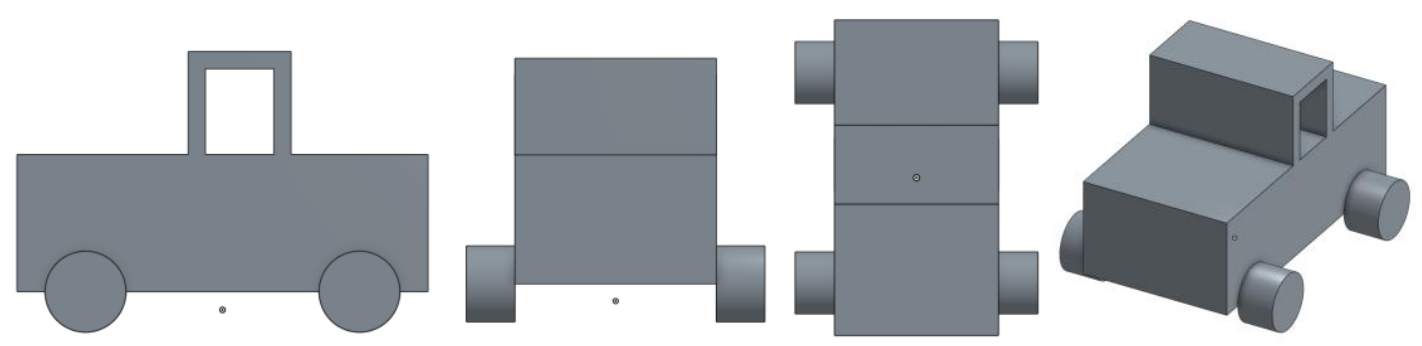

Figure 3. Initial CAD file (ordered left to right) (a) Side view (b) Front view (c) Top view (d) Isometric view

After reviewing the initial CAD file, participants were tasked with a list of 39 changes that were hypothetically gathered from customer review sessions. The goal set forth to our participants was to implement as many changes as possible in 60 minutes. The pair or individual with maximum number of changes was declared a winner and incentivized with a prize in the form of food.

\subsection{Participant profiles and equipment}

Our participant pool consisted of eight mechanical engineering students from MIT at the graduate and senior undergraduate level. It was required that all participants had at least taken a design class at MIT and had used CAD for more than a year. The only exception made to this prerequisite was for $N B-N$, 
the novice participant, who was a mechanical engineering graduate student with limited CAD experience. In total, we recruited eight participants to fulfil the quota required to run all the different scenarios in our study. On average, participants had 1.9 years of CAD experience. The gender distribution of our study pool had equal representation of both male and female participants.

Recruitment was done by emailing the student community and proper consent protocol was followed to share anonymized study data. Participants were instructed not to divulge their identity to each other to avoid any possible biases from knowing each other.

\subsection{Measurements}

On-screen footage was recorded to be used as the primary mode of data capture. In addition, a debrief session was held after the study using a survey questionnaire asking participants to rate various aspects of their study experience and to hear their thoughts on the provided CAD software. All on-screen video footage was processed post experiment and timed data sheets of change implementation were produced. When a participant returned to a previous change later in the experiment, the additional rework time was added to the original change time.

For the purpose of our study, we define value added ratio as the ratio of time spent on CAD modelling tasks versus total study time. A second metric of interest is productivity, defined as total number of CAD changes implemented by a participant. Time based metrics as such have been adapted by other researchers studying collaborative CAD work and provide a good baseline for comparison (Holyoak et al., 2014).

\section{RESULTS AND ANALYSIS}

Results from our study are mostly drawn from quantitative datasets acquired from timestamps of the on-screen footage and consolidating survey responses. In addition, qualitative insights from open response survey questions and observing participant behavior during the study helped us frame an overall conclusion. Images of the final CAD model from the study are shown in Figure 4. As apparent from these images, pairs ended up with more evolved CAD files by the end of our study.

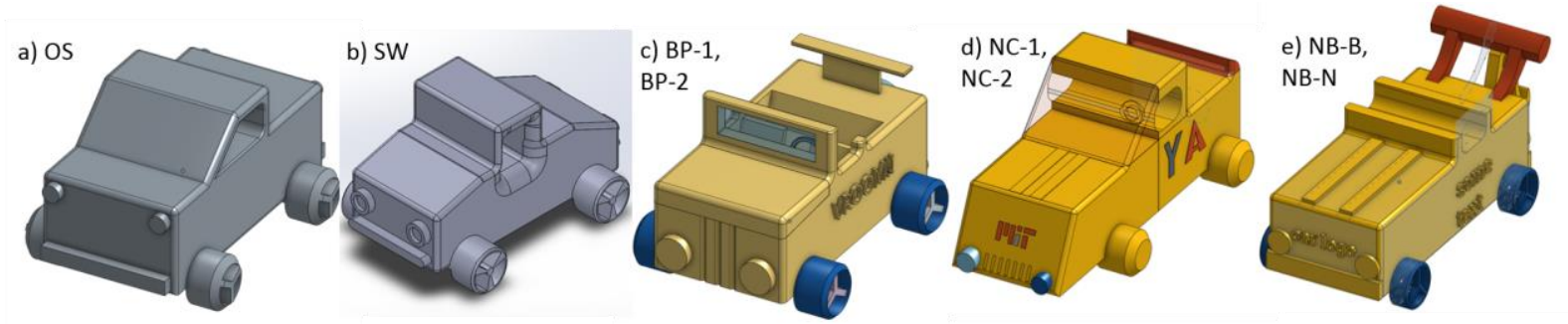

Figure 4. Final CAD files using various working styles (a) OS single (b) SW single

(c) Baseline pair (d) No communication (e) Novice/Baseline

\subsection{Performance analysis (value added ratio and productivity of CAD work)}

To get a finer understanding of the value added ratio of each working style, we aggregated time spent performing non-CAD related activities. All quantitative data and survey findings are summarized in Table 1 below. 
Table 1. Summary of study data (OS: Onshape, SW: SolidWorks, BP: Baseline pair, NC: No communication, NB-B: Baseline participant, NB-N: Novice participant)

\begin{tabular}{|c|c|c|c|c|c|c|c|c|c|}
\hline & & \multicolumn{2}{|c|}{ Individual users } & \multicolumn{2}{|c|}{ Baseline Pair } & \multicolumn{2}{|c|}{ No communication } & \multicolumn{2}{|c|}{ Novice/Baseline } \\
\hline & & OS & SW & BP-1 & BP-2 & NC-1 & NC-2 & NB-B & NB-N \\
\hline & Value added ratio & 0.68 & 0.87 & 0.67 & 0.86 & 0.75 & 0.53 & 0.88 & 0.47 \\
\hline & Productivity (total \# of changes) & 17 & 15 & 17 & 11 & 17 & 7 & 21 & 3 \\
\hline \multirow{5}{*}{ 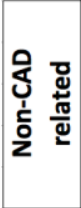 } & Communication (plan/status) (\%) & NA & NA & $5.0 \%$ & $3.8 \%$ & NA & NA & $3.9 \%$ & $9.5 \%$ \\
\hline & Communication (help/advice) (\%) & NA & NA & $1.1 \%$ & $0.6 \%$ & NA & NA & $5.7 \%$ & $9.4 \%$ \\
\hline & Communication (total) (\%) & NA & NA & $6.1 \%$ & $4.3 \%$ & NA & NA & $9.6 \%$ & $18.9 \%$ \\
\hline & Help menu/internet help (\%) & $1.7 \%$ & $3.1 \%$ & $0.0 \%$ & $0.5 \%$ & $0.0 \%$ & $3.0 \%$ & $0.0 \%$ & $1.2 \%$ \\
\hline & Unfinished/aborted changes (\%) & $25.9 \%$ & $5.4 \%$ & $18.5 \%$ & $2.9 \%$ & $22.6 \%$ & $39.5 \%$ & $0.0 \%$ & $31.0 \%$ \\
\hline \multirow{4}{*}{ 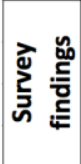 } & Experience (months) & 12 & 12 & 36 & 48 & 36 & 24 & 13 & 1 \\
\hline & Preferred CAD platform & SW & SW & OS & SW & Multiple & SW & Rhino & AutoCAD \\
\hline & Preferred communication & Video & In person & In person & In person & In person & In person & In person & In person \\
\hline & Redo study as? & Individual & Individual & Group & Individual & Individual & Group & Individual & Group \\
\hline
\end{tabular}

\subsubsection{Value added ratio}

Taking aggregates of value added ratio measurements in Table 1, we can ascertain that on average single user CAD participants (0.77) spent more time on CAD work than pairs (0.69). Some of this discrepancy can be explained by the fact that paired participants had to account for additional overheads like spending time to keep up to date with the latest state of the CAD file, communicating with their partners, and planning work together. It is also noteworthy that from amongst the two pairs that could communicate, the Baseline pair seems to rely on communication mostly for planning and status whereas significant portion of the Novice/Baseline pair communication was spent on helping and advising. This finding is consistent with Stone et al. (2017) which shows that successful synchronous CAD teams communicate less. It is also interesting to note that participants did not generally rely on the software help menu and often preferred seeking help from their partners when communication was available. For most participants, unfinished or aborted changes were the dominant factor driving low value added ratio.

Value added ratio was calculated by aggregating all CAD work time for each participant. However, it is important to understand the progression of value added ratio over the span of our study, as plotted in Figure 5. In case of the single user CAD participants, $O S$ has a higher value added ratio until approximately minute 40; after which significant time is lost to unsuccessful attempts which result in a lower time spent towards finished CAD tasks. The similarity of these graphs also attests to the fact that Solidworks and Onshape are similar in their feature set while working on basic CAD tasks. Even though $O S$ and $S W$ worked separately, their results are shown on the same graph for ease of comparison. It is interesting to note that almost all participants operate at the same rate for the first ten minutes with exception of the Novice/Baseline pair.

After the first 10 minutes, all paired working style curves start exhibiting some drifting apart, indicating that the two partners proceed at different rates. This phenomenon could possibly be explained by the inherent history dependency in a CAD file structure wherein each new change is built upon a previous change. It is likely that for our given change list these effects are noticeable after 10 minutes of CAD work. For a CAD session with a value added ratio of 1.0, we should expect to see a horizontal line with $\mathrm{Y}$-axis intercept of 1.0. Participant $B P-1$ and $N B-B$ tend to this ideal state. It is evident that in each of the pairings, we see a leader/follower relationship. This distinction is less clear in the Baseline pair and No communication pairs as they both seem to perform at a similar rate throughout the study, resulting in closer value added ratio. In case of No communication pair, past minute 40 , we begin to see a separation in the curves, potentially due to the lack of communication, and time spent by participants to decipher each other's work.

Looking at the Novice/Baseline pair in Figure 5 (c), we see a large gap between the curves, which could be the result of $N B-B$ 's preference to work mostly by themselves and not leveraging the additional $\mathrm{CAD}$ personnel resource in the environment. This might represent a limitation of synchronous $\mathrm{CAD}$ as being less conducive towards a learner/teacher style collaboration. 


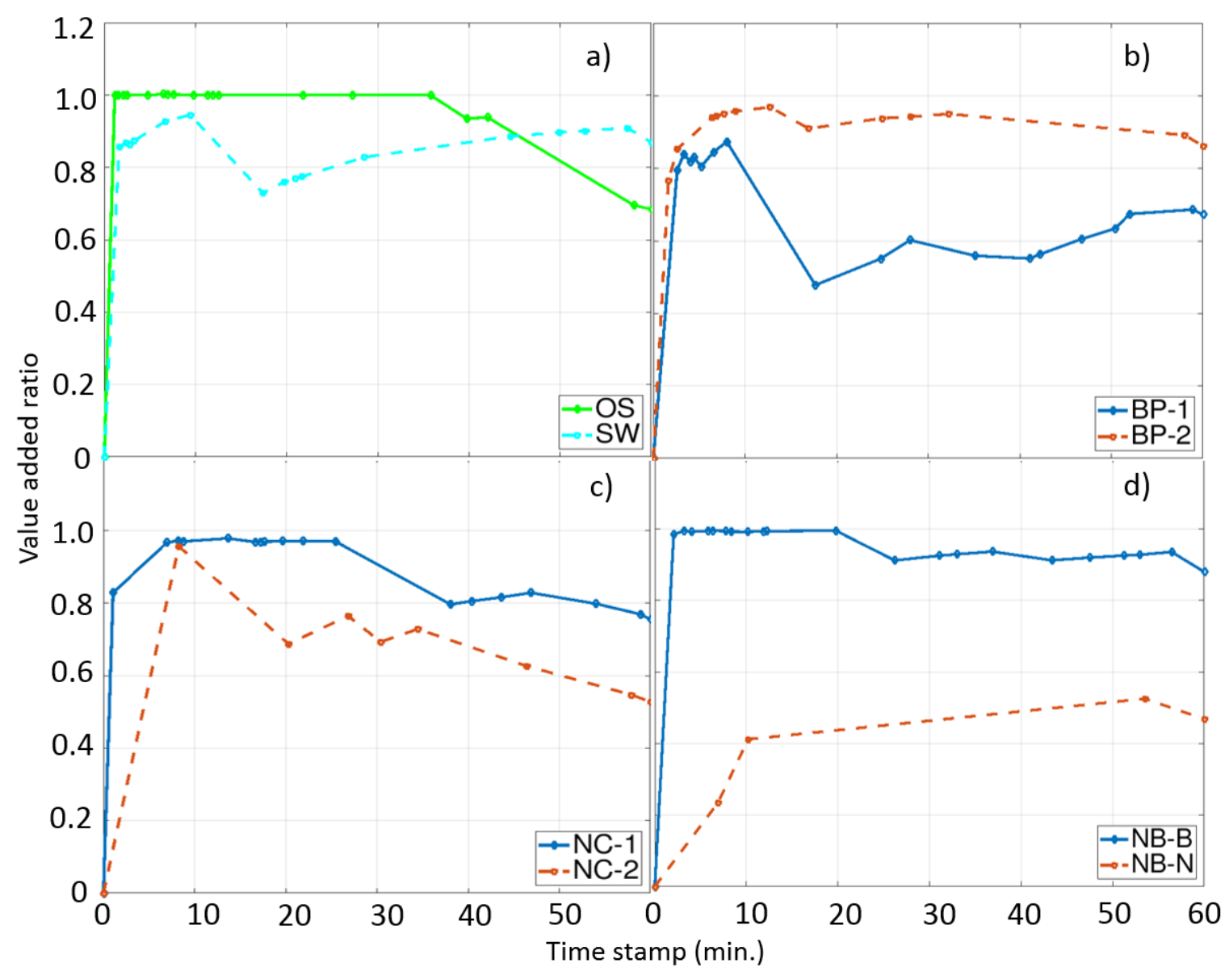

Figure 5. Value added ratio curves: (a) OS/ SW single (b) Baseline pair (c) No communication (d) Novice/Baseline (OS: Onshape, SW: SolidWorks, BP: Baseline pair, NC: No communication, NB-N: Novice participant, NB-B: Baseline participant)

\subsubsection{Productivity}

As can be calculated from Table 1, average productivity for all participants was around 13 changes with a high of 21 for $N B-B$ and a low of 3 for $N B-N$. In Figure 6, we show progression of productivity for each participant over the study time. We see little evidence of correlation between CAD value added ratio and productivity, that is we see no clear matching trend between curves on Figure 5 and Figure 6 for a given participant.

In Figure 6, the rate of increase indicates increase in productivity of our participants. A given point on the area curves in Figure 6 indicates the number of changes accomplished until that point. As can be seen, the single user working style participants, yet again, fared the best in terms of productivity. Their area coverage is minimum and stays consistent. This can be expected as single user CAD participants did not have to work around each other's work. 


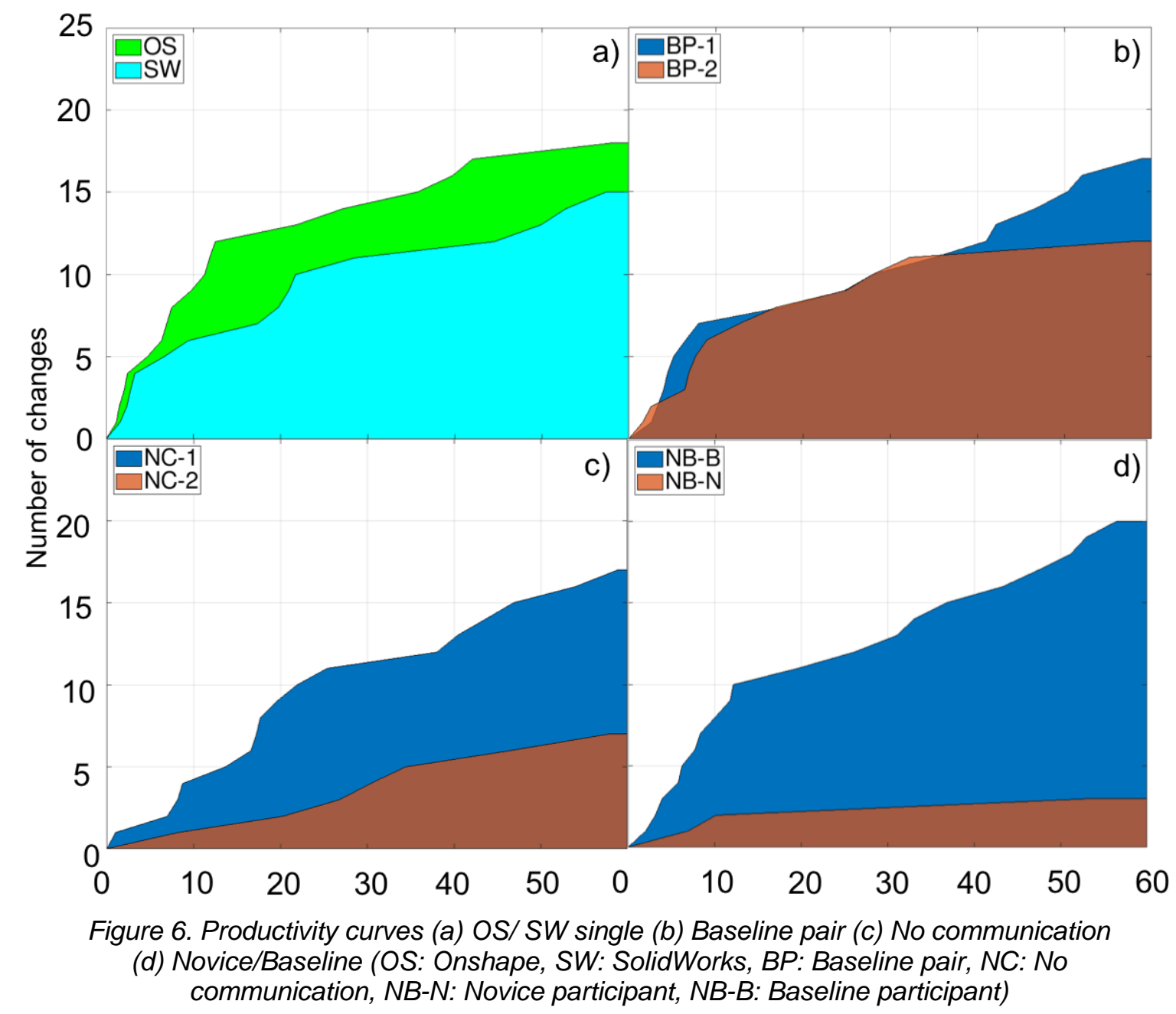

The Baseline pair chart (Figure 6b) is comparable to $O S$ and $S W$ with peaks in the first 10 minutes followed by flatter sections thereafter. We posit that the lack of communication shows its most drastic effect on productivity of the participants, as can be seen in Figure 6c. $N C$-2's productivity is heavily compromised from the beginning and is reduced even more for the second half of the study. This could be attributed to the struggle in establishing awareness of each other's work without the ability to communicate. From Figure $6 \mathrm{c}$, it can be interpreted that $N C-1$ took charge and led the changes whereas $N C-2$ was following and compensating for $\mathrm{NC}-1$ 's work all along. Lastly the Novice/Baseline pair plots are similar to the No communication pair, wherein baseline participant $N B-B$ effectively worked by him/herself. $N C-N$ could not benefit from the collaboration much and ended up accomplishing very little resulting in a low overall productivity for the pair.

\subsubsection{Survey findings}

All participants were given a survey at the end of their CAD study session and their responses are summarized in the bottom section of Table 1. It is noteworthy that everyone except participant $O S$ declared 'in person' communication as their preferred mode of interaction. Participants were also asked to mention a preferred working style if they were to redo the study. Interestingly, the majority of the participants stated that they would work by themselves if they were to redo the study. This might indicate an inherent bias of mechanical engineers toward working in CAD by themselves, but our survey pool is too small to make any statistical claims.

The survey also consisted of open response questions which provided more candid feedback from participants. Some noteworthy responses asking participants to share their opinion on synchronous CAD work were as follows:

"A screen share tool or a way to show my cursor and vice versa will be helpful" - NB-N

"Working in parallel felt a bit frustrating to me" - $B P-2$ 
"I think collaborative cloud-based CAD is a great tool if you can communicate with the other person at all. (ex- establishing a plan in person before CADing separately or via text in the CAD software)." $N C-1$

"CAD is difficult when people are making changes at the same time but it's great to be able to observe other people's work in real time" - $B P-1$

These comments highlight some of the challenges in setting up collaboration in fully synchronous CAD environments and the necessity of design supports.

\section{CONCLUSION AND FUTURE WORK}

\subsection{Conclusion}

Based on our literature survey and learnings from the study, it is evident that synchronous CAD collaboration needs additional scaffolding to be successful. A lack of communication lines or preplanning can lead to loss of value added ratio and productivity. Some of these deficiencies can be traced to the inherent architecture of a CAD model, and history dependence of feature design. By allowing multiple users to work on the same CAD file, challenges around fluency of communication and awareness of others' workspace need to be tackled. Based on our limited participant pool, almost all participants prefer in-person meetings over remote collaboration for the given design task; thus, pointing to the inability of newer platforms to provide a good replacement to traditional communication. This could inhibit a team's shared understanding of the CAD model. In summary, we observed the below findings in our exploratory study:

- Single participants had low non-CAD related time in comparison to pairs, resulting in a higher value added ratio on average. Single working style participants were also more productive than pairs.

- All working styles were noticeably slower after 10mins of study time, which may be due to history dependency or coupled nature of CAD models.

- Baseline pair communicated to plan and update each other. The Novice/Baseline pair used communication for help and advising in addition to planning and updating; leading to an overall higher communication time.

\subsection{Future work}

To make our work comprehensive, it is imperative to conduct a larger study to better understand trends and to establish statistical significance of our findings. The scope of this exploratory study was wide and attempts to test multiple variables at once. In a future iteration of this study, we will reduce the parameter space to probe only a few variables to gain more quantitative insights.

As discussed earlier, studies of collaboration during design have shown that the output is contingent on a number of factors, including information richness, nature of the problem, team composition and more (Martins et al., 2004; Ostergaard and Summers, 2009). Fully-synchronous CAD software provides an appealing instrument with which to explore these factors. An example future study of interest would investigate the creativity of the product enabled by fully-synchronous collaborative design teams when compared to results already discovered for creativity with traditional CAD use and other virtual tools (Chulvi et al., 2017; Häggman et al., 2015; Robertson and Radcliffe, 2009). Another might be a study of team composition, specific to the CAD context and compared to previous findings on collaborative design (McComb et al., 2016). Unused data captured in our study will help us better frame this future work. Communication messages between pairs were archived and will be analyzed. Web camera footage of the designers' faces was also recorded and will be explored in future work.

\section{REFERENCES}

Andreadis, G., Fourtounis, G. and Bouzakis, K.D. (2015), "Collaborative design in the era of cloud computing”, Advances in Engineering Software, Elsevier Ltd, Vol. 81 No. C, pp. 66-72.

Bucciarelli, L. and Kuhn, S. (1997), "Engineering education and engineering practise: Improving the fit", Between Craft and Science: Technical Work in the United States.

Chulvi, V., Mulet, E., Felip, F. and García-García, C. (2017), "The effect of information and communication technologies on creativity in collaborative design", Research in Engineering Design, Vol. 28 No. 1, pp. 7-23. 
Eves, K., Salmon, J., Olsen, J. and Fagergren, F. (2018), “A comparative analysis of computer-aided design team performance with collaboration software”, Computer-Aided Design and Applications, Vol. 4360, pp. $1-12$.

Häggman, A., Tsai, G., Elsen, C., Honda, T. and Yang, M.C. (2015), “Connections Between the Design Tool, Design Attributes, and User Preferences in Early Stage Design”, Journal of Mechanical Design, Vol. 137 No. 7, p. 071101.

Holyoak, V.L., Red, E. and Jensen, G. (2014), "Effective Collaboration through Multi user CAx by Implementing New Methods of Product Specification and Management", Computer-Aided Design and Applications, Vol. 11 No. 5, pp. 560-567.

Li, W.D., Lu, W.F., Fuh, J.Y.H. and Wong, Y.S. (2005), “Collaborative computer-aided design-research and development status”, Computer-Aided Design, Vol. 37 No. 9, pp. 931-940.

Libardi, E.C., Dixon, J.R. and Simmons, M.K. (1988), "Computer environments for the design of mechanical assemblies: A research review”, Engineering with Computers, Vol. 3 No. 3, pp. 121-136.

Martins, L.L., Gilson, L.L. and Maynard, M.T. (2004), "Virtual teams: What do we know and where do we go from here?", Journal of Management, Vol. 30 No. 6, pp. 805-835.

McComb, C., Cagan, J. and Kotovsky, K. (2016), “Optimizing design teams based on problem properties: Computational team simulations and an applied empirical test”, Submitted to Journal of Mechanical Design, Vol. 139 No. April, available at:https://doi.org/10.1115/1.4035793.

Ostergaard, K.J. and Summers, J.D. (2009), "Development of a systematic classification and taxonomy of collaborative design activities", Journal of Engineering Design, Vol. 20 No. 1, pp. 57-81.

Robertson, B.F. and Radcliffe, D.F. (2009), "Impact of CAD tools on creative problem solving in engineering design", CAD Computer Aided Design, Elsevier Ltd, Vol. 41 No. 3, pp. 136-146.

Stone, B., Salmon, J., Eves, K., Killian, M., Wright, L., Oldroyd, J., Gorrell, S., et al. (2017), “A multi-user computer-aided design competition: experimental findings and analysis of team-member dynamics", Journal of Computing and Information Science in Engineering, Vol. 17 No. 3, p. 031003.

Stone, B., Salmon, J.L., Hepworth, A.I., Red, E., Killian, M., La, A., Pedersen, A., et al. (2017), "Methods for determining the optimal number of simultaneous contributors for multi-user CAD parts", ComputerAided Design and Applications, Vol. 14 No. 5, pp. 610-621.

Woo, T. (2014), The Ease of Agile Development in Cloud-Based CAD, Vol. 9, available at: www.aberdeen.com.

Wu, D., Terpenny, J. and Schaefer, D. (2017), "Digital design and manufacturing on the cloud: A review of software and services", Artificial Intelligence for Engineering Design, Analysis and Manufacturing: AIEDAM, Vol. 31 No. 1, pp. 104-118.

\section{ACKNOWLEDGMENTS}

The authors would like to thank our participants and the team at MIT BRL for their time and support in making this work possible. Lastly, we would like to thank the CADLab, Ready Lab, and Ideation community for all the stimulating conversations that influenced our work in a positive way. 